PROCEEDINGS OF THE

AMERICAN MATHEMATICAL SOCIETY

Volume 137, Number 1, January 2009, Pages 339-348

S 0002-9939(08)09490-2

Article electronically published on April 22, 2008

\title{
GEOMETRIC BROWNIAN MOTION WITH DELAY: MEAN SQUARE CHARACTERISATION
}

\author{
JOHN A. D. APPLEBY, XUERONG MAO, AND MARKUS RIEDLE
}

(Communicated by Richard C. Bradley)

\begin{abstract}
A geometric Brownian motion with delay is the solution of a stochastic differential equation where the drift and diffusion coefficients depend linearly on the past of the solution, i.e. a linear stochastic functional differential equation. In this work the asymptotic mean square behaviour of a geometric Brownian motion with delay is completely characterised by a sufficient and necessary condition in terms of the drift and diffusion coefficients.
\end{abstract}

\section{INTRODUCTION}

Geometric Brownian motion is one of the stochastic processes most often used in applications, not least of all in financial mathematics for modelling the dynamics of security prices. More recently, however, modelling the price process by a geometric Brownian motion has been criticised because the past of the volatility is not taken into account.

The geometric Brownian motion is the strong solution of the stochastic differential equation

$$
d X(t)=b X(t) d t+\sigma X(t) d W(t) \quad \text { for } t \geqslant 0,
$$

where $b$ and $\sigma$ are some real constants. If we wish that the dynamics of the process $X$ at time $t$ are to depend on its past, a natural generalisation involves replacing the constants $b$ and $\sigma$ by some linear functionals on an appropriate function space, say the space of continuous functions on a bounded interval. Then we are led by the Riesz representation theorem to the following stochastic differential equation with delay:

$$
d X(t)=\left(\int_{[-\alpha, 0]} X(t+u) \mu(d u)\right) d t+\left(\int_{[-\alpha, 0]} X(t+u) \nu(d u)\right) d W(t)
$$

for all $t \geqslant 0$ and some measures $\mu, \nu$. We call the solution $X$ of this stochastic differential equation geometric Brownian motion with delay and its asymptotic mean

Received by the editors March 23, 2007, and, in revised form, November 15, 2007, and January 11, 2008.

2000 Mathematics Subject Classification. Primary 60H20,60H10, 34K20, 34K50.

Key words and phrases. Stochastic functional differential equations, geometric Brownian motion, mean square stability, renewal equation, variation of constants formula.

The first author was partially funded by an Albert College Fellowship, awarded by Dublin City University's Research Advisory Panel. 
square behavior will be characterised in this article. In contrast to the geometric Brownian motion without delay, no explicit representation of $X$ is known from which the asymptotic behaviour can be inferred directly.

Equation (1) is a stochastic functional differential equation with diffusion coefficient depending on the past. Such equations may exhibit most irregular asymptotic behaviour; see, for example, Mohammed and Scheutzow [8] for the noisy feedback equation. Nevertheless, for a much more general equation than (1) a wide variety of sufficient conditions have been established guaranteeing stability in some sense. An exhaustive list of researchers and papers is not quoted here, but a good selection of such results is collated in Mao [7] and Kolmanovskii and Myshkis [6]. Despite this activity over the last twenty-five years, to the best of our knowledge no sufficient and necessary conditions are known for stability, even for the linear equation (11). By contrast, in this work we are able to find necessary and sufficient conditions which characterise completely the asymptotic behaviour of the solution in the mean square. An interesting by-product of this stability characterisation is the observation that a deterministic solution may transpire to be a solution of the stochastic equation. This feature cannot arise in linear non-delay stochastic equations.

The proof of our stability characterisation relies on the fact that a non-negative functional of the process has expected value which satisfies a deterministic linear renewal equation. The asymptotic behaviour of this functional is characterised by the renewal theorem; once this characterisation has been obtained, it is straightforward to characterise the asymptotic behaviour of the mean square.

\section{Preliminaries}

We first turn our attention to the deterministic delay equation underlying the stochastic differential equation (11). For a fixed constant $\alpha \geqslant 0$ we consider the deterministic linear delay differential equation

$$
\begin{aligned}
\dot{x}(t) & =\int_{[-\alpha, 0]} x(t+u) \mu(d u) \text { for } t \geqslant 0, \\
x(t) & =\varphi(t) \text { for } t \in[-\alpha, 0],
\end{aligned}
$$

for a measure $\mu \neq 0$ in $M=M[-\alpha, 0]$, the space of signed Borel measures on $[-\alpha, 0]$ with the total variation norm $\|\cdot\|_{T V}$. The initial function $\varphi$ is assumed to be in the space $C[-\alpha, 0]:=\{\psi:[-\alpha, 0] \rightarrow \mathbb{R}:$ continuous $\}$. A function $x:[-\alpha, \infty) \rightarrow \mathbb{R}$ is called a solution of (2) if $x$ is continuous on $[-\alpha, \infty)$, its restriction to $[0, \infty)$ is continuously differentiable, and $x$ satisfies the first and second identity of (2) for all $t \geqslant 0$ and $t \in[-\alpha, 0]$, respectively. It is well known that for every $\varphi \in C[-\alpha, 0]$ the problem (2) admits a unique solution $x=x(\cdot, \varphi)$.

The fundamental solution or resolvent of (2) is the unique locally absolutely continuous function $r:[0, \infty) \rightarrow \mathbb{R}$ which satisfies

$$
r(t)=1+\int_{0}^{t} \int_{[\max \{-\alpha,-s\}, 0]} r(s+u) d \mu(u) d s \text { for } t \geqslant 0 .
$$

It plays a role which is analogous to the fundamental system in linear ordinary differential equations and the Green function in partial differential equations. Formally, it is the solution of (2) corresponding to the initial function $\varphi=\mathbb{1}_{\{0\}}$. For later convenience we set $r(u)=0$ for $u \in[-\alpha, 0)$. 
The solution $x(\cdot, \varphi)$ of (2) for an arbitrary initial segment $\varphi$ exists, is unique, and can be represented as

$$
x(t, \varphi)=\varphi(0) r(t)+\int_{[-\alpha, 0]} \int_{s}^{0} r(t+s-u) \varphi(u) d u \mu(d s) \quad \text { for } t \geqslant 0 ;
$$

cf. Diekmann et al. [3, Chapter I].

The asymptotic behaviour of $r$ and hence of $x(\cdot, \varphi)$ relies on the value of

$$
v_{0}(\mu):=\sup \left\{\Re(\lambda): \lambda \in \mathbb{C}, \lambda-\int_{[-\alpha, 0]} e^{\lambda s} \mu(d s)=0\right\},
$$

where $\Re(z)$ denotes the real part of a complex number $z$. We summarize some conditions on the asymptotic behavior of the fundamental solution $r$ in the following lemma:

Lemma 2.1. For the fundamental solution $r$ the following are equivalent:
(a) $v_{0}(\mu)<0$;
(b) $r(t) \rightarrow 0$ for $t \rightarrow \infty$;
(c) $r \in L^{1}\left(\mathbb{R}_{+}\right)$;
(d) $r \in L^{2}\left(\mathbb{R}_{+}\right)$.

Proof. The equivalences of (a), (b) and (c) are established by Theorems 3.3.5 and 3.3.17 in Gripenberg et al. [5]. Obviously, (b) and (c) imply (d). Conversely, the equation

$$
\dot{r}(t)=\int_{[-\alpha, 0]} r(t+u) \mu(d u) \text { for } t \geqslant 0
$$

implies, by Hölder's inequality, that $\dot{r}$ is in $L^{2}\left(\mathbb{R}_{+}\right)$. For $f(t)=r^{2}(t)$ it follows by Hölder's inequality that $\dot{f} \in L^{1}\left(\mathbb{R}_{+}\right)$and therefore $f(t) \rightarrow 0$ for $t \rightarrow \infty$ as $f \in L^{1}\left(\mathbb{R}_{+}\right)$.

For every $\gamma>v_{0}(\mu)$ we have $r(t)=o(\exp (\gamma t))$ for $t \rightarrow \infty$ by [5, Thm. 7.2.1]. Consequently, if $r$ tends to zero the decay is exponentially fast with a rate greater than $v_{0}(\mu)$. By (3) every solution $x(\cdot, \varphi)$ inherits the asymptotic behavior of $r$; that is, $x(t, \varphi)=\mathcal{O}(\exp (\gamma t))$ for $t \rightarrow \infty$ and all $\gamma>v_{0}(\mu)$.

Let us introduce equivalent notation for (2). For a function $x:[-\alpha, \infty) \rightarrow \mathbb{R}$ we define the segment of $x$ at time $t \geqslant 0$ by the function

$$
x_{t}:[-\alpha, 0] \rightarrow \mathbb{R}, \quad x_{t}(u):=x(t+u) .
$$

If we equip the space $C[-\alpha, 0]$ of continuous functions with the supremum norm, then the Riesz representation theorem guarantees that every continuous functional $F: C[-\alpha, 0] \rightarrow \mathbb{R}$ is of the form

$$
F(\psi)=\int_{[-\alpha, 0]} \psi(u) \mu(d u)
$$

for a measure $\mu \in M$. Hence, we will write (2) in the form

$$
\dot{x}(t)=F\left(x_{t}\right) \quad \text { for } t \geqslant 0, \quad x_{0}=\varphi
$$

and assume $F$ to be a continuous and linear functional on $C[-\alpha, 0]$. 
Let us fix a complete probability space $(\Omega, \mathcal{F}, P)$ with a filtration $\left(\mathcal{F}_{t}\right)_{t \geqslant 0}$ satisfying the usual conditions and let $(W(t): t \geqslant 0)$ be a Wiener process on this space. We study the following stochastic differential equation with time delay:

$$
\begin{aligned}
d X(t) & =F\left(X_{t}\right) d t+G\left(X_{t}\right) d W(t) \quad \text { for } t \geqslant 0, \\
X(u) & =\varphi(u) \quad \text { for } u \in[-\alpha, 0],
\end{aligned}
$$

where $F$ and $G$ are continuous and linear functionals on $C[-\alpha, 0]$ for a constant $\alpha \geqslant 0$. As before, we can write the functional $G$ in the form

$$
G(\psi)=\int_{[-\alpha, 0]} \psi(u) \nu(d u) \quad \text { for all } \psi \in C[-\alpha, 0]
$$

and for a measure $\nu \in M$. We note that assuming the same domain $[-\alpha, 0]$ for the arguments of the functionals $F$ and $G$ does not involve any restriction or loss of generality.

For every $\varphi \in C[-\alpha, 0]$ there exists a unique, adapted strong solution $(X(t, \varphi)$ : $t \geqslant-\alpha$ ) with finite second moments of (5) (cf., e.g., Mao [7, Thm. 5.2.6]). The dependence of the solutions on the initial condition $\varphi$ is neglected in our notation in what follows; that is, we will write $x(t)=x(t, \varphi)$ and $X(t)=X(t, \varphi)$ for the solutions of (2) and (5) respectively.

By Reiß et al. [9, Lemma 6.1] the solution $(X(t): t \geqslant-\alpha)$ of (5) obeys a variation of constants formula

$$
X(t)= \begin{cases}x(t)+\int_{0}^{t} r(t-s) G\left(X_{s}\right) d W(s), & t \geqslant 0, \\ \varphi(u), & u \in[-\alpha, 0],\end{cases}
$$

where $r$ is the fundamental solution of (2). It is to be noted that this equation does not supply an explicit form of the solution.

\section{Stability}

The asymptotic behaviour of the solution $X$ relies on the stochastic convolution integral arising in the variation of constants formula (6). Let us define

$$
Y(t):=G\left(X_{t}\right) \quad \text { for } t \geqslant 0,
$$

such that the stochastic convolution integral is the convolution of the stochastic process $Y=(Y(t): t \geqslant 0)$ and the fundamental solution $r$. The following result shows that the function $t \mapsto E|Y(t)|^{2}$ satisfies a linear convolution integral equation.

Theorem 3.1. Let $(X(t): t \geqslant-\alpha)$ be the solution of (5). Then we have for all $t \geqslant 0$,

$$
E|X(t)|^{2}=|x(t)|^{2}+\int_{0}^{t} r^{2}(t-s) E|Y(s)|^{2} d s,
$$

where $Y$, defined by (17), obeys for all $t \geqslant 0$ :

$$
E|Y(t)|^{2}=G^{2}\left(x_{t}\right)+\int_{0}^{t} G^{2}\left(r_{t-s}\right) E|Y(s)|^{2} d s .
$$

Proof. The variation of constants formula (6) and Itô's isometry imply the first assertion. 
Using again the variation of constants formula we obtain by Fubini's theorem for stochastic integrals for $t \in[0, \alpha]$ :

$$
\begin{aligned}
E|Y(t)|^{2}= & E\left|G\left(X_{t}\right)\right|^{2} \\
= & E\left|\int_{[-\alpha,-t)} X_{t}(u) \nu(d u)+\int_{[-t, 0]} X_{t}(u) \nu(d u)\right|^{2} \\
=E & \mid \int_{[-\alpha,-t)} \varphi(t+u) \nu(d u)+\int_{[-t, 0]} x(t+u) \nu(d u) \\
& +\left.\int_{[-t, 0]}\left(\int_{0}^{t+u} r(t+u-s) Y(s) d W(s)\right) \nu(d u)\right|^{2} \\
=E & \left|G\left(x_{t}\right)+\int_{0}^{t}\left(\int_{[s-t, 0]} r(t+u-s) \nu(d u)\right) Y(s) d W(s)\right|^{2} \\
= & \left|G\left(x_{t}\right)\right|^{2}+\int_{0}^{t} G^{2}\left(r_{t-s}\right) E|Y(s)|^{2} d s,
\end{aligned}
$$

where we used in the last line $r(u)=0$ for $u<0$. Setting $\nu([a, b))=0$ for all $a \leqslant b \leqslant-\alpha$ enables us to enlarge the integration domain for $G$ such that we can write also for $t \geqslant \alpha$ :

$$
E|Y(t)|^{2}=E\left|G\left(x_{t}\right)+\int_{[-t, 0]}\left(\int_{0}^{t+u} r(t+u-s) Y(s) d W(s)\right) \nu(d u)\right|^{2} .
$$

We can proceed as above to verify that equation (9) is also satisfied for $t \geqslant \alpha$.

We next turn to stating and proving our first stability result. In it, the hypothesis $r \in L^{2}\left(\mathbb{R}_{+}\right)$is employed. We remark that this assumption is necessary if $E|X(t, \varphi)|^{2} \rightarrow 0$ as $t \rightarrow \infty$ for all solutions $X(\cdot, \varphi)$ for every $\varphi \in C[-\alpha, 0]$. To see this, note by (8) that $E|X(t, \varphi)|^{2}$ cannot tend to zero as $t \rightarrow \infty$ if $x(t, \varphi)$ does not tend to zero. But $x(t, \varphi)$ tends to zero for all $\varphi \in C[-\alpha, 0]$ if and only if $v_{0}(\mu)<0$, which might be seen by the spectral decomposition of $C[-\alpha, 0]$; see [2, Thm. 7.2.1].

The function $s \mapsto G\left(r_{s}\right)$, which we denote by $G\left(r_{\bullet}\right)$, is square integrable if $r \in L^{2}\left(\mathbb{R}_{+}\right)$and its norm in $L^{2}\left(\mathbb{R}_{+}\right)$is given by

$$
\left\|G\left(r_{\bullet}\right)\right\|_{L^{2}\left(\mathbb{R}_{+}\right)}=\left(\int_{0}^{\infty}\left(G\left(r_{s}\right)\right)^{2} d s\right)^{1 / 2} .
$$

This quantity allows us to characterise the asymptotic behaviour of the solution for (5):

Theorem 3.2. If the fundamental solution $r$ is in $L^{2}\left(\mathbb{R}_{+}\right)$, then the solution $(X(t)$ : $t \geqslant-\alpha)$ of (5) obeys the following trichotomy:

(a) If $\left\|G\left(r_{\bullet}\right)\right\|_{L^{2}\left(\mathbb{R}_{+}\right)}<1$, then there exists $\kappa>0$ such that

$$
\lim _{t \rightarrow \infty} e^{\kappa t} E|X(t)|^{2}=0
$$


(b) If $\left\|G\left(r_{\bullet}\right)\right\|_{L^{2}\left(\mathbb{R}_{+}\right)}=1$, then

$$
\lim _{t \rightarrow \infty} E|X(t)|^{2}=\frac{\left(\int_{0}^{\infty} G^{2}\left(x_{s}\right) d s\right)\left(\int_{0}^{\infty} r^{2}(s) d s\right)}{\int_{0}^{\infty} s G^{2}\left(r_{s}\right) d s}<\infty
$$

(c) If $\left\|G\left(r_{\bullet}\right)\right\|_{L^{2}\left(\mathbb{R}_{+}\right)}>1$, then there exists $\kappa>0$ such that

$$
\lim _{t \rightarrow \infty} e^{-\kappa t} E|X(t)|^{2}=\frac{\left(\int_{0}^{\infty} e^{-\kappa s} G^{2}\left(x_{s}\right) d s\right)\left(\int_{0}^{\infty} e^{-\kappa s} r^{2}(s) d s\right)}{\int_{0}^{\infty} s e^{-\kappa s} G^{2}\left(r_{s}\right) d s}<\infty .
$$

Proof. Let us introduce the following functions and measures for $t \geqslant 0$ :

$$
\begin{aligned}
y(t):=E|Y(t)|^{2}, & f(t) & :=G^{2}\left(x_{t}\right), \\
g(t):=G^{2}\left(r_{t}\right), & \zeta(d t) & :=g(t) d t .
\end{aligned}
$$

Then we can rewrite (9) as the renewal equation

$$
y(t)=f(t)+\int_{0}^{t} y(t-s) \zeta(d s) \text { for all } t \geqslant 0
$$

and the three cases (a) to (c) correspond to whether the renewal equation (10) is defective, proper or excessive.

Moreover, we introduce the notation $h_{(\lambda)}(t):=e^{\lambda t} h(t)$ for any function $h: \mathbb{R}_{+} \rightarrow$ $\mathbb{R}$ and $\zeta_{(\lambda)}(d t):=e^{\lambda t} \zeta(d t)$ for $\lambda \in \mathbb{R}$. The equation (10) can be rewritten as

$$
y_{(\lambda)}(t)=f_{(\lambda)}(t)+\int_{0}^{t} y_{(\lambda)}(t-s) \zeta_{(\lambda)}(d s) \text { for all } t \geqslant 0
$$

and all $\lambda \in \mathbb{R}$. We analyse the asymptotic behaviour of $y_{(\lambda)}$ by the renewal Theorems 3.1.4 and 3.1.5 in Alsmeyer [1. If $\zeta_{(\lambda)}\left(\mathbb{R}_{+}\right)<1$ and $f_{(\lambda)}(t) \rightarrow 0$ for $t \rightarrow \infty$, we obtain by [1, Thm. 3.1.4] that

$$
\lim _{t \rightarrow \infty} y_{(\lambda)}(t)=0 \text {. }
$$

In the proper case, that is, $\zeta_{(\lambda)}\left(\mathbb{R}_{+}\right)=1$, it is sufficient for the application of the renewal theorem that the function $f_{(\lambda)}$ is in $\left(L^{1} \cap L^{\infty}\right)\left(\mathbb{R}_{+}\right)$and satisfies $f_{(\lambda)}(t) \rightarrow 0$ for $t \rightarrow \infty$ because the measure $\zeta$ is absolutely continuous with respect to the Lebesgue measure. Then the renewal theorem [1, Thm. 3.1.5] yields

$$
\lim _{t \rightarrow \infty} y_{(\lambda)}(t)=\left(m\left(\zeta_{(\lambda)}\right)\right)^{-1} \int_{0}^{\infty} f_{(\lambda)}(s) d s,
$$

where $m\left(\zeta_{(\lambda)}\right):=\int_{\mathbb{R}_{+}} t e^{\lambda t} \zeta(d t)$.

By Lemma 2.1 and a remark after this lemma we have $r(t)=o(\exp (-\gamma t))$ and $x(t)=o(\exp (-\gamma t))$ for all $\gamma \in\left(0,-v_{0}(\mu)\right)$. Now fix such a $\gamma$.

Let us first assume in case (a) that $\left\|G\left(r_{\bullet}\right)\right\|_{L^{2}\left(\mathbb{R}_{+}\right)}=0$. Then we have $G\left(r_{s}\right)=0$ for all $s \geqslant 0$, and by (9) we have $y(t)=G^{2}\left(x_{t}\right)=o(\exp (-2 \gamma t))$, and we can proceed as below.

In case (a) the assumption $0<\left\|G\left(r_{\bullet}\right)\right\|_{L^{2}\left(\mathbb{R}_{+}\right)}<1$ yields that there exists $\vartheta>0$ with

$$
\zeta_{(\vartheta)}\left(\mathbb{R}_{+}\right)=\int_{0}^{\infty} e^{\vartheta s} \zeta(d s)=1
$$


In the case $\vartheta \leqslant 2 \gamma$ we have $f_{(\vartheta)}(t) \rightarrow 0$ and the renewal theorem (13) implies

$$
\lim _{t \rightarrow \infty} y_{(\vartheta)}(t)=\left(m\left(\zeta_{(\vartheta)}\right)\right)^{-1} \int_{0}^{\infty} f_{(\vartheta)}(s) d s .
$$

Note that $m\left(\zeta_{(\vartheta)}\right)$ is non-zero because of (14) and the integral term is finite because of the exponential decay of $x$.

In the other case $2 \gamma<\vartheta$ we have $f_{(2 \gamma)}(t) \rightarrow 0$ and $\zeta_{(2 \gamma)}\left(\mathbb{R}_{+}\right)<1$. Therefore, the renewal theorem (12) yields

$$
\lim _{t \rightarrow \infty} y_{(2 \gamma)}(t)=0 .
$$

Consequently, we have together in case (a) that $y(t)=o(\exp (-\kappa t))$ for all $\kappa<$ $(2 \gamma \wedge \vartheta)$, which leads to

$$
\int_{0}^{t} r^{2}(s) y(t-s) d s=o(\exp (-\kappa t)) .
$$

Because equation (8) can be written as

$$
E|X(t)|^{2}=x^{2}(t)+\int_{0}^{t} r^{2}(s) y(t-s) d s,
$$

the proof is complete.

In case (b), the renewal theorem (13) guarantees that

$$
\lim _{t \rightarrow \infty} y(t)=(m(\zeta))^{-1} \int_{0}^{\infty} f(s) d s
$$

with $m(\zeta):=m\left(\zeta_{(0)}\right)$. Note that $m(\zeta)$ is finite as $r$ tends to zero exponentially fast and it is non-zero because $\int_{0}^{\infty} G^{2}\left(r_{s}\right) d s=1$. By equation (15) we obtain by dominated convergence

$$
\begin{aligned}
\lim _{t \rightarrow \infty} E|X(t)|^{2} & =\lim _{t \rightarrow \infty} \int_{0}^{\infty} r^{2}(s) \mathbb{1}_{[0, t]} y(t-s) d s \\
& =\frac{1}{m(\zeta)}\left(\int_{0}^{\infty} f(s) d s\right)\left(\int_{0}^{\infty} r^{2}(s) d s\right) .
\end{aligned}
$$

In case (c), because $\zeta$ is a finite measure, there exists a unique $\vartheta<0$ in case (c) such that $\zeta_{(\vartheta)}\left(\mathbb{R}_{+}\right)=\int_{\mathbb{R}_{+}} e^{\vartheta s} \zeta(d s)=1$. Furthermore, as before, $m\left(\zeta_{(\vartheta)}\right)$ is non-zero and finite. The renewal theorem (13) implies

$$
\lim _{t \rightarrow \infty} y_{(\vartheta)}(t)=\left(m\left(\zeta_{(\vartheta)}\right)\right)^{-1} \int_{0}^{\infty} e^{\vartheta s} f(s) d s
$$

Finally, from (8) we have

$$
e^{\vartheta t} E|X(t)|^{2}=e^{\vartheta t}|x(t)|^{2}+\int_{0}^{t} e^{\vartheta s} r^{2}(s) e^{\vartheta(t-s)} y(t-s) d s,
$$

and so, because of the exponential decay of $r$, we conclude

$$
\lim _{t \rightarrow \infty} e^{\vartheta t} E|X(t)|^{2}=\frac{1}{m\left(\zeta_{\vartheta}\right)}\left(\int_{0}^{\infty} e^{\vartheta s} f(s) d s\right)\left(\int_{0}^{\infty} e^{\vartheta s} r^{2}(s) d s\right) .
$$

Note that in the proof we give an upper bound for the decay rate $\kappa$ in case (a) of Theorem 3.2 .

Alsmeyer [1] contains a treatment of the renewal equation which covers equations with measures. Similar results may be found in Feller [4]. 
As a corollary of Theorem 3.2 we obtain an equivalence between the asymptotic behaviour of the mean square of the solution $X$ and a condition on the fundamental solution $r$ and the diffusion coefficient $G$. Naturally, this requires that the solution $X$ does not reduce to the solution of the deterministic equation (2), for in this case $X$ would not provide any information on the diffusion coefficient. We argue below that this situation may occur and must be excluded in the next corollary. This will also be illustrated presently in an example.

Corollary 3.3. Let the fundamental solution $r$ be in $L^{2}\left(\mathbb{R}_{+}\right)$and assume that no version of the solution $X=X(\cdot, \varphi)$ of (5) coincides with the deterministic solution $x=x(\cdot, \varphi)$ of (2). Then we have the following:

$$
\lim _{t \rightarrow \infty} E|X(t)|^{2}= \begin{cases}0 & \Longleftrightarrow\left\|G\left(r_{\bullet}\right)\right\|_{L^{2}\left(\mathbb{R}_{+}\right)}<1, \\ c>0 & \Longleftrightarrow\left\|G\left(r_{\bullet}\right)\right\|_{L^{2}\left(\mathbb{R}_{+}\right)}=1, \\ \infty & \Longleftrightarrow\left\|G\left(r_{\bullet}\right)\right\|_{L^{2}\left(\mathbb{R}_{+}\right)}>1 .\end{cases}
$$

Proof. We have to show that the constants in Theorem 3.2 in (b) and (c) describing the limiting behavior of $X$ are non-zero, which is equivalent to

$$
\int_{0}^{\infty} G^{2}\left(x_{s}(\cdot, \varphi)\right) d s \neq 0 .
$$

Because $s \mapsto x_{s}(\cdot, \varphi)$ and $G$ are continuous operators, equation (16) does not hold if and only if $G\left(x_{t}(\cdot, \varphi)\right)=0$ for all $t \geqslant 0$. In this case $x(\cdot, \varphi)$ would also be a solution of the stochastic equation (5), which contradicts our assumption because of the uniqueness of the solution of (5).

Remark 3.4. If the solution $x(\cdot, \varphi)$ of the deterministic equation (2) also solves the stochastic equation (5), then it follows by taking expectation and Itô's isometry that

$$
G\left(x_{t}(\cdot, \varphi)\right)=0 \quad \text { for all } t \geqslant 0 .
$$

Conversely, condition (17) implies that a version of the solution $X(\cdot, \varphi)$ of (5) coincides with $x(\cdot, \varphi)$.

Thus, a sufficient condition for the trichotomy only relying on the initial condition $\varphi$ is $G(\varphi) \neq 0$.

A more abstract point of view shows that equation (17) holds true if a generalized eigenspace $N$ of the deterministic equation (2) is a subset of the $\operatorname{kernel} \operatorname{ker}(G)$ of the diffusion coefficient $G$. Then, for every $\varphi \in N$ the segment $x_{t}(\cdot, \varphi)$ is in $N$ and consequently in $\operatorname{ker}(G)$, so that (17) is satisfied. For details on eigenspaces and related results for equation (2), we refer the reader to Diekmann et al. [3] and Hale and Lunel [2]. A concrete example is given below.

We emphasise that the foregoing situation in which a deterministic solution may solve a non-trivial linear stochastic differential equation is a very specific feature of stochastic functional differential equations and cannot occur in linear stochastic ordinary differential equations.

Example 3.5. Let us consider the solution $(X(t): t \geqslant 0)$ of the simple equation

$$
d X(t)=b X(t) d t+(c X(t)+d X(t-\alpha)) d W(t) \quad \text { for } t \geqslant 0,
$$


with $b<0, c, d \in \mathbb{R}$ and $\alpha>0$. For the condition in Theorem 3.2, as $r(t)=0$ for $t<0$, we calculate

$$
\begin{aligned}
\left\|G\left(r_{\bullet}\right)\right\|_{L^{2}\left(\mathbb{R}_{+}\right)}^{2} & =\int_{0}^{\infty} G^{2}\left(r_{s}\right) d s \\
& =c^{2} \int_{0}^{\infty} r^{2}(s) d s+d^{2} \int_{\alpha}^{\infty} r^{2}(s-\alpha) d s+2 c d \int_{\alpha}^{\infty} r(s) r(s-\alpha) d s \\
& =\frac{1}{-2 b}\left(c^{2}+d^{2}+2 c d e^{b \alpha}\right) .
\end{aligned}
$$

By using results on deterministic linear difference equations we obtain for a continuous function $y:[-\alpha, \infty) \rightarrow \mathbb{R}$ :

$$
\begin{aligned}
G\left(y_{t}\right)=0 \quad \text { for all } t \geqslant 0 & \Longleftrightarrow c y(t)+d y(t-\alpha)=0 \quad \text { for all } t \geqslant 0 \\
& \Longleftrightarrow y(t)=y(0) e^{\gamma t \quad \text { for all } t \geqslant-\alpha \quad \text { and } \quad c d<0}
\end{aligned}
$$

with $\gamma:=\frac{1}{\alpha} \ln \frac{-d}{c}$. In the case when $b \neq \gamma$ and $c d<0$, we obtain that for every initial condition $\varphi$ the solution $X=X(\cdot, \varphi)$ obeys

$$
\lim _{t \rightarrow \infty} E|X(t)|^{2}=0 \Longleftrightarrow c^{2}+d^{2}+2 c d e^{b \alpha}<-2 b .
$$

In the case when $b<0$ and $c d \geqslant 0$, the equivalence (19) also holds.

In the non-delay case $d=0$ the solution $X$ is the geometric Brownian motion and (19) reproduces the well-known and easily calculated fact that $E|X(t)|^{2} \rightarrow 0$ if and only if $c^{2}<-2 b$. In the pure delay case $c=0$ and $d \neq 0$ we find that $d^{2}<-2 b$ is necessary and sufficient to guarantee $E|X(t)|^{2} \rightarrow 0$. However, although the condition on the noise intensities is the same as for geometric Brownian motion, the rate of decay to zero is different.

If $c \neq 0$ and $d \neq 0$, then the dependence of the stability region on the drift coefficient $b$ is described by $b<b_{0}<0$ where $b_{0}$ is the largest real root of

$$
c^{2}+d^{2}+2 c d e^{b_{0} \alpha}+2 b_{0}=0 .
$$

In particular, we observe that while the stability region for (18) is symmetric in $c$ and $d$, it is not symmetric in the sign of $c d$.

We finish by pointing out that equation (18) provides an example of the situation already mentioned in Remark 3.4] in which a solution of the deterministic equation (2) is also a solution of the stochastic equation (5). To see this take, for example, $c=-e, d=1$ and $b=\gamma$ for some $\alpha \geqslant 1$. Then, for $\varphi(u)=e^{\gamma u}, u \in[-\alpha, 0]$, the solution $x(t, \varphi)=e^{\gamma t}, t \geqslant 0$, satisfies $G\left(x_{t}(\cdot, \varphi)\right)=0$ for all $t \geqslant 0$. Thus, $x(\cdot, \varphi)$ is also a solution of the stochastic equation and $x(t, \varphi) \rightarrow 0$ for $t \rightarrow \infty$ even though $\left\|G\left(r_{\bullet}\right)\right\|_{L^{2}\left(\mathbb{R}_{+}\right)}^{2}=\frac{\alpha}{2}\left(e^{2}-1\right)>1$.

\section{REFERENCES}

[1] G. Alsmeyer, Erneuerungstheorie. Analyse stochastischer Regenerationsschemata, Stuttgart: B. G. Teubner, 1991. MR.1119301 (92f:60148)

[2] J. K. Hale and S. M. Verduyn Lunel, Introduction to Functional Differential Equations, New York: Springer-Verlag, 1993. MR.1243878 (94m:34169)

[3] O. Diekmann, S. A. van Gils, S. M. Verduyn Lunel and H.-O. Walther, Delay Equations. Functional, Complex, and Nonlinear Analysis, New York: Springer-Verlag, 1995. MR1345150 (97a:34001)

[4] W. Feller, An Introduction to Probability Theory and Its Applications, Volume II, New York: Wiley, 1971. MR0270403(42:5292) 
[5] G. Gripenberg, S.-O. Londen and O. Staffans, Volterra Integral and Functional Equations, Cambridge: Cambridge University Press, 1990. MR 1050319 (91c:45003)

[6] V. Kolmanovskii and A. Myshkis, Introduction to the Theory and Applications of Functional Differential Equations, Dordrecht: Kluwer Academic Publishers, 1999. MR1680144 (2000c:34164)

[7] X. Mao, Stochastic Differential Equations and Their Applications, Chichester: Horwood Publishing, 1997. MR.1475218 (2000g:60099)

[8] S.-E. A. Mohammed and M. K. R. Scheutzow, Lyapunov exponents of linear stochastic functional differential equations. II: Examples and case studies, Ann. Probab., 25, 3, 1210-1240, 1997. MR1457617 (99e:60134)

[9] M. Reiß, M. Riedle and O. van Gaans, On Émery's inequality and a variation-of-constants formula, Stochastic Anal. and Appl., 25, 2, 353-379, 2007. MR2303092 (2008b:60138)

School of Mathematical Sciences, Dublin City University, Dublin 9, Ireland

E-mail address: john.appleby@dcu.ie

Department of Statistical and Modelling Science, Strathclyde University, Glasgow, UNITED KINGDOM

E-mail address: xuerong@stams.strath.ac.uk

School of Mathematics, The University of Manchester, Oxford Road, Manchester M13 9PL, United Kingdom

E-mail address: markus.riedle@manchester.ac.uk 